\title{
Phase-space characterization of complexity in quantum many-body dynamics
}

\author{
Vinitha Balachandran, ${ }^{1}$ Giuliano Benenti, ${ }^{2,3}$ Giulio Casati, ${ }^{2,3,4}$ and Jiangbin Gong ${ }^{1,5}$ \\ ${ }^{1}$ Department of Physics and Center for Computational Science and Engineering, \\ National University of Singapore, Singapore 117542 \\ ${ }^{2}$ CNISM, CNR-INFM \& Center for Nonlinear and Complex Systems, \\ Università degli Studi dell'Insubria, Via Valleggio 11, 22100 Como, Italy \\ ${ }^{3}$ Istituto Nazionale di Fisica Nucleare, Sezione di Milano, via Celoria 16, 20133 Milano, Italy \\ ${ }^{4}$ Centre for Quantum Technologies, National University of Singapore, Singapore 117543 \\ ${ }^{5}$ NUS Graduate School for Integrative Sciences and Engineering, Singapore 117597
}

(Dated: November 5, 2018)

\begin{abstract}
We propose a phase-space Wigner harmonics entropy measure for many-body quantum dynamical complexity. This measure, which reduces to the well known measure of complexity in classical systems and which is valid for both pure and mixed states in single-particle and many-body systems, takes into account the combined role of chaos and entanglement in the realm of quantum mechanics. The effectiveness of the measure is illustrated in the example of the Ising chain in a homogeneous tilted magnetic field. We provide numerical evidence that the multipartite entanglement generation leads to a linear increase of entropy until saturation in both integrable and chaotic regimes, so that in both cases the number of harmonics of the Wigner function grows exponentially with time. The entropy growth rate can be used to detect quantum phase transitions. The proposed entropy measure can also distinguish between integrable and chaotic many-body dynamics by means of the size of long term fluctuations which become smaller when quantum chaos sets in.
\end{abstract}

PACS numbers: 05.45.Mt, 03.67.Mn, 05.30.-d

\section{INTRODUCTION}

Understanding the dynamics of quantum systems is a challenging task of immense importance in a variety of fields including condensed matter physics and quantum information science. Quantum dynamical complexity refers to the lack of a simple description of the evolution of a quantum system. From a computational perspective, it implies the inevitable loss of predictability of system evolution using classical simulation. In manybody interacting quantum systems, complexity can be attributed to non-integrability or to the tensor product structure of the Hilbert space. Hence, quantum chaos and entanglement have deep implications in characterizing quantum many-body dynamical complexity.

In classical physics, it is very well known that there exists a direct correlation between chaos and complexity. Classically chaotic systems are characterized by exponentially diverging nearby trajectories, with a rate determined by the Lyapunov exponent. Complexity then arises from the fact that the orbits of such deterministic systems are completely random and unpredictable with positive algorithmic complexity [1]. In quantum mechanics, trajectories in standard treatments are forbidden by the Heisenberg uncertainty principle and therefore the above notion of complexity cannot be directly translated to quantum systems.

However, the phase-space approach can be equally used for both classical and quantum mechanics. In the context of classical systems, it has been shown that the second moment of the Fourier components of the classical distribution function grows linearly for an integrable system while it grows exponentially for a chaotic sys- tem, with a rate determined by the Lyapunov exponent characterizing the local exponential instability. Thus, the growth rate of the second moment of Fourier components (harmonics) is a good measure of the complexity of classical dynamics 22. In a similar way, for single-particle quantum systems the second moment of harmonics of the Wigner distribution function of a quantum state, pure or mixed, is a measure of quantum complexity [2, 3]. Note that in quantum systems with few degrees of freedom an exponential growth of the number of harmonics is possible only up to the Ehrenfest time scale, after which the growth is at most linear [3]. Moreover, the number of harmonics of the Wigner function can be used to detect, in the time domain, the crossover from integrability to chaos [4].

For quantum many-body systems the situation is more complicated. First note that quantum dynamical entropies, which generalize the Komologrov-Sinai entropy to quantum dynamical systems, can be positive even for integrable dynamics [5]. This behavior may appear, at least at first sight, somehow surprising since in classical dynamics positive Komologorov-Sinai entropy implies chaos. Another interesting property is that, as shown in Ref. [6], the rank of the matrix product operator representation of the pure quantum states in the timedependent density-matrix renormalization group, typically grows exponentially even for integrable system with finite number of particles. This inefficiency of the classical simulation of many-body quantum dynamics can be attributed to entanglement and is consistent with the linear growth of the entanglement block entropy for integrable spin chains [7].

Several very interesting definitions of quantum com- 
plexity have been proposed, e.g., see Ref. [5] and references therein. On the other hand, to the best of our knowledge none of them satisfies all the following requirements, which a notion of complexity should possess in order to be both meaningful and practically useful:

(i) To provide a unified description of both one- and many-body dynamics;

(ii) To reproduce at the classical limit the well-known notion of classical complexity based on the local exponential instability of chaotic dynamics;

(iii) To be applicable to both pure and mixed states;

(iv) To be practically useful, that is, convenient for numerical investigations.

The purpose of the present paper is to propose a notion of complexity that fulfills the above criteria. By extending previous investigations [2-4] to many-body quantum dynamics, we propose the number of harmonics of the Wigner function as a suitable measure of complexity of a quantum state. Indeed, as the phase-space formulation of quantum dynamics can be directly generalized to many-body systems, the harmonics of the Wigner function seem to be very promising in quantifying the complexity of many-body quantum systems as well. Hence, in this paper, we introduce a Wigner harmonics entropy measure of complexity and then illustrate its usefulness by means of numerical simulations carried on a paradigmatic spin-chain model, the Ising chain in a tilted magnetic field. We will show that the entropy grows linearly until saturation in both integrable and chaotic regimes, so that in both cases the number of harmonics of the Wigner function grows exponentially with time. We will provide numerical evidence that this growth must be attributed to multipartite entanglement generation. Our results demonstrate that the growth rate can be used also to detect quantum phase transitions. Finally, the proposed entropy measure can also distinguish between integrable and chaotic many-body dynamics, by means of the size of long term fluctuations, which become smaller when a transition to chaos occurs.

This paper is organized as follows. In Sec. III, we define our phase-space quantum complexity measure, based on the harmonics of the Wigner function. The working of such measure is illustrated in the dynamics of a manybody spin-chain model, introduced in Sec. [II and investigated in detail in Sec. IV] Finally, our conclusions are drawn in Sec. V

\section{HARMONICS OF THE WIGNER FUNCTION}

The phase-space representation of quantum mechanics is a very enlightening approach as it allows a direct comparison between quantum and classical dynamics. In particular, the complexity of a quantum state or of a classical distribution function can be measured by the richness of their phase space structure.

In the quantum case, the phase-space approach to complexity is particularly convenient for systems whose Hamiltonian can be written in terms of a set of bosonic creation-annihilation operators:

$$
\begin{gathered}
\hat{H}\left(\hat{a}_{1}^{\dagger}, \ldots, \hat{a}_{N}^{\dagger}, \hat{a}_{1}, \ldots, \hat{a}_{N} ; t\right) \equiv \hat{H}^{(0)}\left(\hat{n}_{1}, \ldots, \hat{n}_{N}\right) \\
+\hat{H}^{(1)}\left(\hat{a}_{1}^{\dagger}, \ldots, \hat{a}_{N}^{\dagger}, \hat{a}_{1}, \ldots, \hat{a}_{N} ; t\right)
\end{gathered}
$$

with $\left[\hat{a}_{i}, \hat{a}_{j}\right]=\left[\hat{a}_{i}^{\dagger}, \hat{a}_{j}^{\dagger}\right]=0,\left[\hat{a}_{i}^{\dagger}, \hat{a}_{j}\right]=\delta_{i j}$, and the number operators $\hat{n}_{i}=\hat{a}_{i}^{\dagger} \hat{a}_{i}$.

We will use the method of $c$-number $\boldsymbol{\alpha}$-phase space borrowed from quantum optics (see for example Ref. [8]). The Wigner function $W\left(\boldsymbol{\alpha}, \boldsymbol{\alpha}^{*} ; t\right)$ of a state $\hat{\rho}(t)$ is defined by

$$
\begin{gathered}
W\left(\boldsymbol{\alpha}, \boldsymbol{\alpha}^{*} ; t\right)=\frac{1}{\pi^{2 N} \hbar^{N}} \int d^{2} \boldsymbol{\eta} \exp \left(\frac{\boldsymbol{\eta}^{*} \cdot \boldsymbol{\alpha}}{\sqrt{\hbar}}-\frac{\boldsymbol{\eta} \cdot \boldsymbol{\alpha}^{*}}{\sqrt{\hbar}}\right) \\
\times \operatorname{Tr}[\hat{\rho}(t) \hat{D}(\boldsymbol{\eta})]
\end{gathered}
$$

where $\boldsymbol{\eta}=\left(\eta_{1}, \ldots, \eta_{N}\right)$ and $\boldsymbol{\alpha}=\left(\alpha_{1}, \ldots, \alpha_{N}\right)$ are $N$ dimensional complex variables, the integration runs over the complex $\eta_{i}$-planes for $i=1, \ldots, N$, the displacement operator

$$
\hat{D}(\boldsymbol{\eta})=\exp \left[\sum_{i=1}^{N}\left(\eta_{i} \hat{a}_{i}^{\dagger}-\eta_{i}^{*} \hat{a}_{i}\right)\right]
$$

and the coherent states

$$
|\boldsymbol{\alpha}\rangle=\left|\alpha_{1} \alpha_{2} \ldots \alpha_{N}\right\rangle=\hat{D}\left(\frac{\boldsymbol{\alpha}}{\sqrt{\hbar}}\right)|00 \ldots 0\rangle,
$$

with $\left|\alpha_{i}\right\rangle$ being eigenstate of the annihilation operator $\hat{a}_{i}$, i.e., $\hat{a}_{i}\left|\alpha_{i}\right\rangle=\frac{\alpha_{i}}{\sqrt{\hbar}}\left|\alpha_{i}\right\rangle$, and $|00 \ldots 0\rangle$ being the vacuum state. We define the harmonic's amplitudes $W_{\mathbf{m}}(\mathbf{I} ; t)$ of the Wigner function by the $N$-dimensional Fourier expansion

$$
W\left(\boldsymbol{\alpha}, \boldsymbol{\alpha}^{*} ; t\right)=\frac{1}{\pi^{N}} \sum_{\mathbf{m}} W_{\mathbf{m}}(\mathbf{I} ; t) e^{i \mathbf{m} \cdot \boldsymbol{\theta}},
$$

where $\mathbf{m}, \mathbf{I}, \boldsymbol{\theta}$ are $N$-dimensional vectors, whose components $I_{k} \geq 0,0 \leq \theta_{k}<2 \pi$ are defined by the relations $\alpha_{k}=\sqrt{I_{k}} e^{-i \theta_{k}}, \bar{k}=1, \ldots, N$. Here $I_{k}$ and $\theta_{k}$ can be regarded as our quantum phase space variables, analogous to the action and angle variables in the classical phase space. Note that $W_{-\mathbf{m}}=W_{\mathbf{m}}^{*}$. The Wigner function's normalization condition $\int d^{2} \boldsymbol{\alpha} W\left(\boldsymbol{\alpha}, \boldsymbol{\alpha}^{*} ; t\right)=1$ simply implies that $\int d \mathbf{I} W_{\mathbf{0}}(\mathbf{I} ; t)=1$, while there are no restrictions on $W_{\mathbf{m}}$ when $\mathbf{m} \neq \mathbf{0}$.

In Refs. [3, 4], the number of harmonics of the Wigner function was estimated by $\sqrt{\left\langle\mathbf{m}^{2}\right\rangle_{t}}$, with $\left\langle\mathbf{m}^{2}\right\rangle_{t}$ being the second moment of the harmonics distribution:

$$
\left\langle\mathbf{m}^{2}\right\rangle_{t}=\sum_{\mathbf{m}} \mathbf{m}^{2} \mathcal{W}_{\mathbf{m}}(t)
$$


where

$$
\mathcal{W}_{\mathbf{m}}(t) \equiv \frac{\int d \mathbf{I}\left|W_{\mathbf{m}}(\mathbf{I} ; t)\right|^{2}}{\sum_{\mathbf{m}} \int d \mathbf{I}\left|W_{\mathbf{m}}(\mathbf{I} ; t)\right|^{2}}
$$

The harmonics distribution $\mathcal{W}_{\mathbf{m}}$ is normalized, $\sum_{\mathbf{m}} \mathcal{W}_{\mathbf{m}}=1$. For one-body systems, the second moment $\left\langle\mathbf{m}^{2}\right\rangle_{t}$ provides a reliable estimate of the number of harmonics in a generic chaotic case [3] and is able to distinguish, in the semiclassical region, between integrable and chaotic regimes [4]. In the first case, $\sqrt{\left\langle\mathbf{m}^{2}\right\rangle_{t}}$ grows linearly in time, in the latter exponentially. On the other hand, we expect that the number of harmonics always captures the complexity of motion, including the case of many-body systems without classical analogue. Hence, we propose as a complexity measure the entropy

$$
S(t)=-\sum_{m_{1}, \ldots, m_{N} \geq 0} \mathcal{W}_{\mathbf{m}}(t) \ln \left[\mathcal{W}_{\mathbf{m}}(t)\right]
$$

where the sum over $\mathbf{m}$ is limited to $m_{1}, \ldots, m_{N} \geq 0$ since harmonics $\mathcal{W}_{\mathbf{m}}$ and $\mathcal{W}_{-\mathbf{m}}$ are not independent but trivially related by the relation $\mathcal{W}_{\mathbf{m}}=\mathcal{W}_{-\mathbf{m}}$ (note that the same limitation must now be taken in Eq. (7) in order to properly normalize the distribution $\mathcal{W}_{\mathbf{m}}$ ). The number of harmonics of a generic state $\hat{\rho}(t)$ can therefore be measured by $\exp [S(t)]$. For the models discussed in this paper both the second moment $\left\langle\mathbf{m}^{2}\right\rangle$ and the entropy $S$ provide qualitatively the same results $[9]$.

The main computational advantage of the above $c$ number $\boldsymbol{\alpha}$-phase space approach is that the Wigner function's harmonics $\mathcal{W}_{\mathrm{m}}$ can be computed very conveniently from the density matrix written in the basis of the eigenvectors $|\mathbf{n}\rangle=\left|n_{1} \ldots n_{N}\right\rangle$ of the unperturbed Hamiltonian $\hat{H}^{(0)}$. Indeed, using the well-known matrix elements of the displacement operator [10],

$\left\langle n_{i}+m_{i}\left|\hat{D}\left(\eta_{i}\right)\right| n_{i}\right\rangle=\sqrt{\frac{n_{i} !}{\left(n_{i}+m_{i}\right) !}} \eta_{i}^{m_{i}} e^{-\frac{1}{2}\left|\eta_{i}\right|^{2}} L_{n_{i}}^{m_{i}}\left(\left|\eta_{i}\right|^{2}\right)$,

$\left(n_{i}, m_{i} \geq 0, i=1, \ldots, N\right)$, where $L_{n_{i}}^{m_{i}}(x)$ is a Laguerre polynomial, the $\boldsymbol{\eta}$-integration in Eq. (2) can be carried out explicitly. After that, using the orthogonality and completeness properties of the Laguerre polynomials along the lines of Ref. [3], we can express the Wigner harmonics $W_{\mathbf{m}}(\mathbf{I} ; t)$ in terms of the matrix elements $\langle\mathbf{n}+\mathbf{m}|\hat{\rho}(t)| \mathbf{n}\rangle$ and finally obtain

$$
\mathcal{W}_{\mathbf{m}}(t)=\frac{\sum_{\mathbf{n}}|\langle\mathbf{n}+\mathbf{m}|\hat{\rho}(t)| \mathbf{n}\rangle|^{2}}{\sum_{m_{1}, \ldots, m_{N} \geq 0} \sum_{\mathbf{n}}|\langle\mathbf{n}+\mathbf{m}|\hat{\rho}(t)| \mathbf{n}\rangle|^{2}} .
$$

Finally, we point out that our approach remains valid also for classical systems, provided the Wigner function is substituted by the classical phase-space distribution function in the $\alpha_{k}$-coordinates, with $\alpha_{k}=\sqrt{I_{k}} e^{-i \theta_{k}}$, $\left\{I_{k}, \theta_{k}\right\}$ being a set of action-angle variables for the unperturbed, integrable Hamiltonian $H_{0}$.

\section{THE MODEL}

In order to investigate the working of our complexity measure, we consider, as an illustrative example, the Ising chain of $N$ spins in a tilted magnetic field. The Hamiltonian reads

$$
\hat{H}=J \sum_{i} \hat{\sigma}_{i}^{z} \hat{\sigma}_{i+1}^{z}+\sum_{i}\left[h_{x} \hat{\sigma}_{i}^{x}+h_{z} \hat{\sigma}_{i}^{z}\right]
$$

where $J$ is the spin-spin coupling constant, $\hat{\sigma}_{i}^{\alpha}$ are the Pauli operators for the $i$-th spin, and $h_{x}, h_{z}$ are the field amplitudes along $x$ and $z$ directions, respectively. We set $\hbar=J=1$. This chain is in general non-integrable, except for the two integrable limits $h_{x}=0$ or $h_{z}=0$. The integrable model $h_{z}=0$ corresponds to the Ising model in a transverse field and exhibits a quantum phase transition at $J=h_{x}$ [11].

Using the Schwinger boson representation [12], the above spin Hamiltonian is mapped onto an interacting boson Hamiltonian. Each spin operator $\hat{\sigma}_{i}$ at the site $i$ is replaced by two Schwinger bosons, $\hat{a}_{i}$ and $\hat{b}_{i}$, corresponding to spin up $\{\uparrow\}$ and down $\{\downarrow\}$. The spin operators can be represented as follows:

$$
\begin{array}{r}
\hat{\sigma}_{i}^{z}=\hat{a}_{i}^{\dagger} \hat{a}_{i}-\hat{b}_{i}^{\dagger} \hat{b}_{i}, \\
\hat{\sigma}_{i}^{+}=\hat{a}_{i}^{\dagger} \hat{b}_{i}, \text { and } \hat{\sigma}_{i}^{-}=\hat{b}_{i}^{\dagger} \hat{a}_{i},
\end{array}
$$

where $\hat{\sigma}^{ \pm}=\frac{1}{2}\left(\hat{\sigma}^{x} \pm i \hat{\sigma}^{y}\right)$. Since we have spin- $1 / 2$ particles, the physical subspace is singled out by the constraints $n_{a i}+n_{b i}=1(i=1, \ldots, N)$, where $n_{a i}$ and $n_{b i}$ denote the number of up and down spins at site $i$ $\left(\hat{n}_{a i}=\hat{a}_{i}^{\dagger} \hat{a}_{i}, \hat{n}_{b i}=\hat{b}_{i}^{\dagger} \hat{b}_{i}\right)$. Now, Eq. (11) takes the form

$$
\begin{aligned}
\hat{H}= & J \sum_{i=1}^{N-1}\left(\hat{a}_{i}^{\dagger} \hat{a}_{i}-\hat{b}_{i}^{\dagger} \hat{b}_{i}\right)\left(\hat{a}_{i+1}^{\dagger} \hat{a}_{i+1}-\hat{b}_{i+1}^{\dagger} \hat{b}_{i+1}\right) \\
& +\sum_{i=1}^{N}\left[h_{x}\left(\hat{a}_{i}^{\dagger} \hat{b}_{i}+\hat{b}_{i}^{\dagger} \hat{a}_{i}\right)+h_{z}\left(\hat{a}_{i}^{\dagger} \hat{a}_{i}-\hat{b}_{i}^{\dagger} \hat{b}_{i}\right)\right] .
\end{aligned}
$$

As the Hamiltonian is now expressed in terms of a set of bosonic creation-annihilation operators, it then follows that the above explained phase-space approach can be used to probe the dynamical complexity of the spin chain.

For a chain of $N$ spins, $\mathbf{n}$ and $\mathbf{m}$ in Eq. (10) are $2 N$ dimensional vectors,

$$
\begin{aligned}
\mathbf{n} & =\left(n_{a 1}, n_{b 1}, n_{a 2}, n_{b 2}, \ldots n_{a N}, n_{b N}\right), \\
\mathbf{m} & =\left(m_{a 1}, m_{b 1}, m_{a 2}, m_{b 2}, \ldots m_{a N}, m_{b N}\right),
\end{aligned}
$$

where the first subscript refers to the spin type and second refers to the spin site. The possible values of $n$ 's are 0 and 1 with the constraint $n_{a i}+n_{b i}=1$. Similarly, the possible values $m$ 's can take are $-1,0$ and 1 with the constraint $m_{a i}+m_{b i}=0$ to remain on the physical subspace. Indeed, the following cases are possible: (i) $m_{a i}=-1$, $m_{b i}=1$, corresponding to the transition of the $i$-th spin from up to down, (ii) $m_{a i}=1, m_{b i}=-1$ (transition of 
the $i$-th spin from down to up), and (iii) $m_{a i}=m_{b i}=0$ (no transition for the $i$-th spin). Due to the trivial relation $\mathcal{W}_{-\mathbf{m}}=\mathcal{W}_{\mathbf{m}}$, we limit the summation (8) to $m_{a i} \geq 0$ (and, consequently, $m_{b i} \leq 0$ ), for $i=1, \ldots, N$, in order to take into account independent terms only. That is, for a chain of $N$ spins, only $2^{N}$ values of the $\mathcal{W}_{\mathbf{m}}$ are independent and are considered for calculations. Note that the maximum possible value of the entropy measure $S(t)$ is $N \ln 2$. This value is reached when maximum mixing occurs so that all the harmonics are equally distributed.

\section{PHASE-SPACE CHARACTERIZATION OF COMPLEXITY}

\section{A. Initial Growth of $S(t)$}

In this section, we study in detail the time evolution of the entropy $S(t)$ at small times. The initial state is chosen to be a pure state with all spins pointing downward in the $z$ direction, i.e., $\left|\Psi_{\text {in }}\right\rangle=|\downarrow \downarrow \ldots \downarrow\rangle$. From the definition of Wigner harmonics $\mathcal{W}_{\mathbf{m}}(t)$ in Eq. (10), it is clear that only the $\mathbf{m}=(0,0, \ldots 0)$ harmonics component is excited, the initial number of harmonics is equal to unity and hence $S(t=0)=0$.

To relate the growth rate of the harmonics to the complexity of the dynamics as in Ref. [4], first we consider the short time behavior of the entropy measure $S(t)$ when the system undergoes a transition to quantum chaos as detected by a change of Poisson to Wigner distribution in the level statistics. To that end, let us rewrite the Hamiltonian $\hat{H}$ as in Eq. (1) with

$$
\hat{H}^{(0)}=J \sum_{i} \hat{\sigma}_{i}^{z} \hat{\sigma}_{i+1}^{z}+\sum_{i} h_{z} \hat{\sigma}_{i}^{z} ; \quad \hat{H}^{(1)}=\sum_{i} h_{x} \hat{\sigma}_{i}^{x} .
$$

Here $\hat{H}^{(0)}$ is the integrable Hamiltonian and $\hat{H}^{(1)}$ represents the perturbation to the chain induced by an external transverse field. As the perturbation is increased, a transition to Wigner-type level statistics and hence quantum chaos occurs. In particular, for $h_{x}=h_{z}=J$ the system can be considered as fully chaotic [6] .

The initial state is an eigenstate of the unperturbed integrable Hamiltonian $\hat{H}^{(0)}$. Hence with zero perturbation $\left(h_{x}=0\right)$, there is no evolution and $S(t)$ remains zero at all times. However, by adding a small perturbation to the system i.e., a small transverse field $h_{x}$, the initial state is no longer an eigenstate of the Hamiltonian $\hat{H}$ and transitions to many other states occur. Then, besides the zeroth harmonics i.e., $\mathbf{m}=(0,0, \ldots 0)$, higher harmonics are also excited and the entropy $S(t)$ increases. Smaller the transverse field, less complex is the dynamical evolution and therefore a lower value of the growth rate for entropy $S(t)$ is obtained. This is clearly seen from our results in Fig. 1(a). For instance, with $h_{x}=0.2$, the chain is near the integrable regime and $S(t)$ is about 1.0 at time $t=1.0$. By contrast, in the chaotic regime with $h_{x}=0.9, S(t)$ increases to 6.3 . Interestingly, for cases (a)

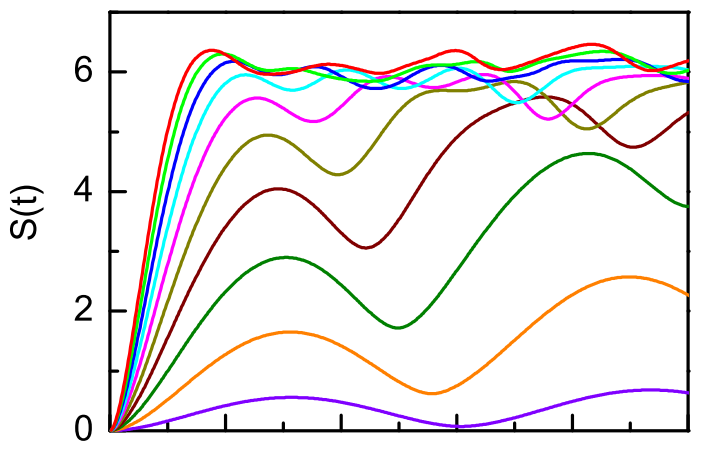

(b)

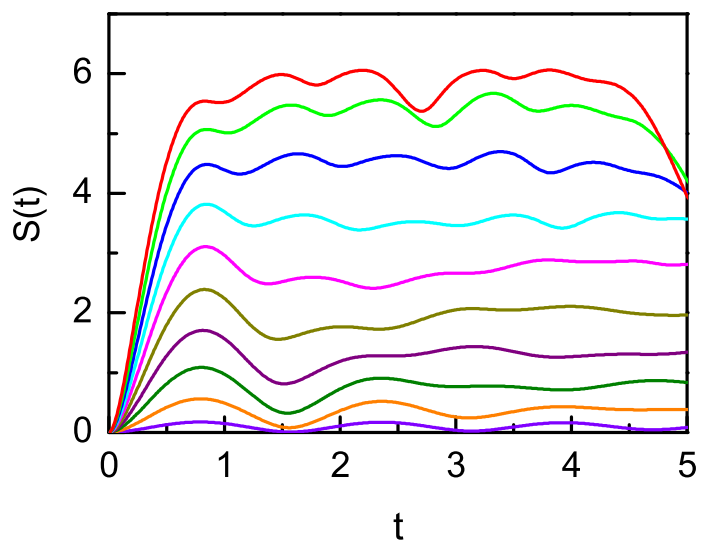

FIG. 1: (color online) Time dependence of the entropy measure $S(t)$ for a chain of 10 spins with (a) non-integrable Hamiltonian as given in Eq. (15) with a longitudinal field $h_{z}=1.0$ and (b) integrable Hamiltonian as given in Eq. (16). Curves from top to bottom correspond to transverse field $h_{x}=1.0$ to 0.1 in decreasing steps of 0.1. Note that during an initial time window $S(t)$ is clearly seen to grow linearly with time, implying the exponential growth of the number of harmonics in both (a) and (b), for a sufficiently large $h_{x}$. All the parameters mentioned here, and in the other figures, are dimensionless (we set $\hbar=J=1$ ).

with a sufficiently strong perturbation, $S(t)$ is seen to increase linearly with time within a time window $(t<0.6)$. Such linear increase of $S(t)$ implies an exponential growth of the number of harmonics.

Consider then another situation for the Ising chain, with the magnetic field applied in the transverse direction only:

$$
\hat{H}^{(0)}=J \sum_{i} \hat{\sigma}_{i}^{z} \hat{\sigma}_{i+1}^{z} ; \quad \hat{H}^{(1)}=\sum_{i} h_{x} \hat{\sigma}_{i}^{x} .
$$

Note that this Hamiltonian is integrable for all values of the perturbation $h_{x}$ [13]. On the basis of previous findings in few-body problems [2, 4], we might expect a linear increase of the number of harmonics, corresponding to a logarithmic growth of $S(t)$. On the contrary, as shown 
(a)

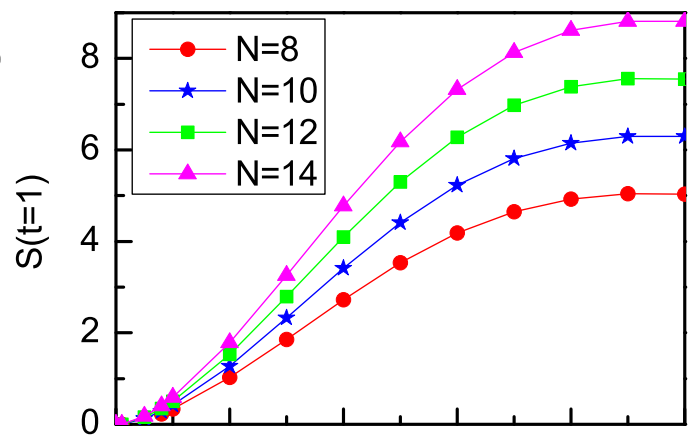

(b)

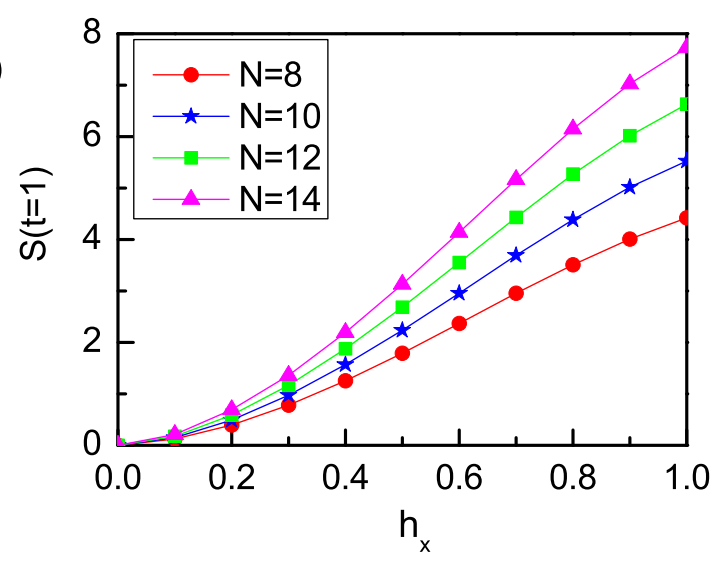

FIG. 2: (color online) Dependence of entropy $S(t)$ at time $t=1$ on the strength of the external perturbation $h_{x}$ for different chain length $N$ for (a) a non-integrable spin chain and (b) an integrable spin chain. For both cases and for sufficiently large $h_{x}$, it is seen that $S(t=1)$ scales linearly with $N$.

in Fig. 1(b) the short time behavior of $S(t)$ is linear. A comparison between Fig. 1(a) and Fig. 1(b) clearly shows that one cannot distinguish between a chaotic and an integrable many-body system by solely examining the initial growth of the number of Wigner harmonics.

To gain more insights, we next study how the initial linear growth rate of $S(t)$ depends on the number $N$ of spins in the chain, for both the non-integrable model (15) and the integrable model (16). In particular, we vary $N$ from $N=8$ to $N=14$. Figure 22(a) shows the value of $S(t)$ at a fixed time $t=1$ as a function of $h_{x}$, for the non-integrable model with four different values of $N$. Note that $S(t=1)$ can be understood as the average entropy production rate for $t \in[0,1]$. It can be observed that $S(t=1)$ scales with $N$ linearly. For example, for $h_{x}=1.0, S(t=1)$ increases by a constant value $(\approx 1)$ as $N$ increases in steps of two. Interestingly, as shown in Fig. 2(b), exactly the same behavior is observed for the integrable model. This further strengthens our early finding that the initial growth of the number of Wigner harmonics is qualitatively the same for non- integrable and integrable spin chains. We must therefore seek an underlying mechanism to account for this somewhat counter-intuitive behavior of many-body quantum systems.

\section{B. Wigner Harmonics and Entanglement}

A source of quantum complexity in many-body systems is the entanglement due to the interaction between the different constituent parts. Recent studies indicated that some measure of the entanglement entropy can also grow linearly with time 7]. We will therefore inquire whether or not the lack of distinction between integrable and non-integrable models shown above is related to the generation of multipartite entanglement.

To quantify the extent of multipartite entanglement generated in a spin chain, we adopt the multipartite entanglement measure used in Ref. [14]. Specifically, the system under consideration is partitioned into two subsystems $A$ and $B$, made up of $n_{A}$ and $n_{B}$ spins, respectively. The participation number $N_{A B}$, defined as the reciprocal of the purity of one of the two subsystems, i.e.,

$$
N_{A B}=\frac{1}{\operatorname{Tr}\left[\hat{\rho}_{A}^{2}\right]},
$$

accounts for the bipartite entanglement between $A$ and $B$. Here, $\hat{\rho}_{A}$ is the reduced density matrix of subsystem $A$. The physical meaning of $N_{A B}$ is that it effectively counts the relevant terms in the Schmidt decomposition of the total wavefunction into the sum of direct products of wavefunctions of the two subsystems. The mean value of $N_{A B}$, averaged over all possible partitions, quantifies the degree of multipartite entanglement in the system, while its variance measures how well the entanglement is distributed. For systems of large size $N>>1$, the statistical weight of unbalanced partitions becomes negligible [14 and hence only balanced partitions are considered here.

In Fig. 3 we present the time dependence of the mean value of the participation number $\left\langle N_{A B}\right\rangle$, starting from the same initial state as before, i.e., $\left|\Psi_{\text {in }}\right\rangle=|\downarrow \downarrow \ldots \downarrow\rangle$, for both integrable and non-integrable models. The initial state is not entangled and hence $\left\langle N_{A B}\right\rangle(t=0)$ is given by its minimum value: unity. Entanglement is then generated by the dynamical evolution of the spin chain and hence $\left\langle N_{A B}\right\rangle$ increases. Remarkably, after a short time interval $(t<0.4)$ and for a sufficiently large value of $h_{x},\left\langle N_{A B}\right\rangle$ reaches the saturation value almost exponentially fast, for both the integrable and non-integrable models. Though the production of entanglement is somewhat slower for the integrable chain as compared to the non-integrable case, an exponential-like fast increase of $\left\langle N_{A B}\right\rangle$ is seen in both situations. To visualize this more clearly, we plot an exponential fit for $h_{x}=0.8$ in both panels of Fig. 3. These results indicate that we can as- 
(a)

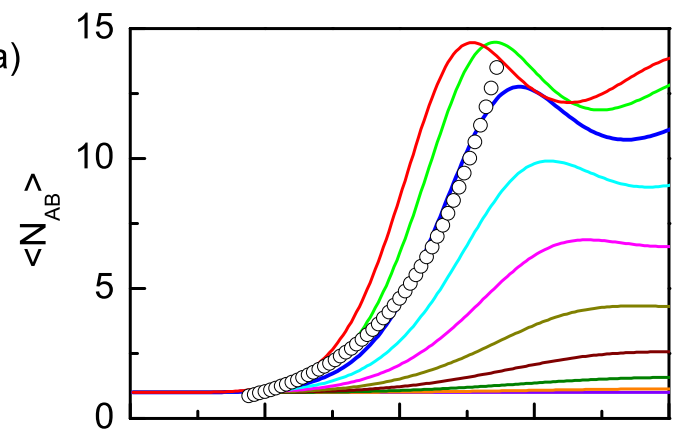

(b)

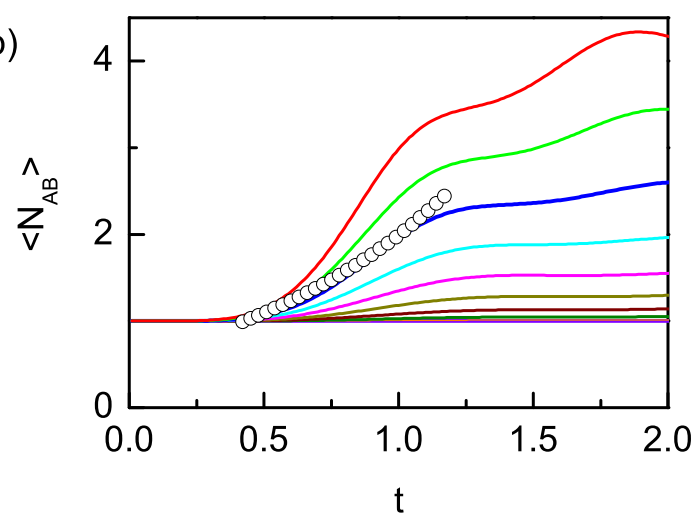

(a)

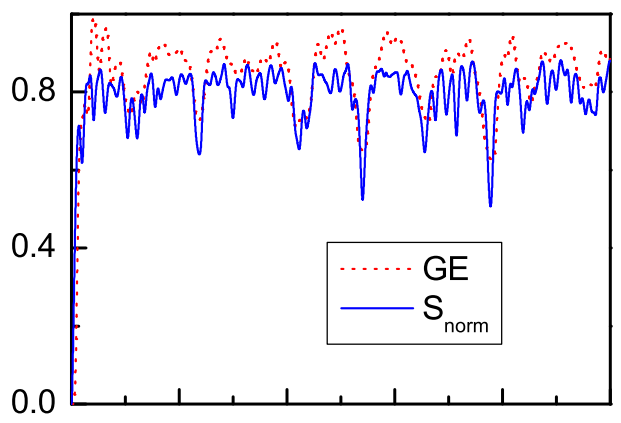

(b)

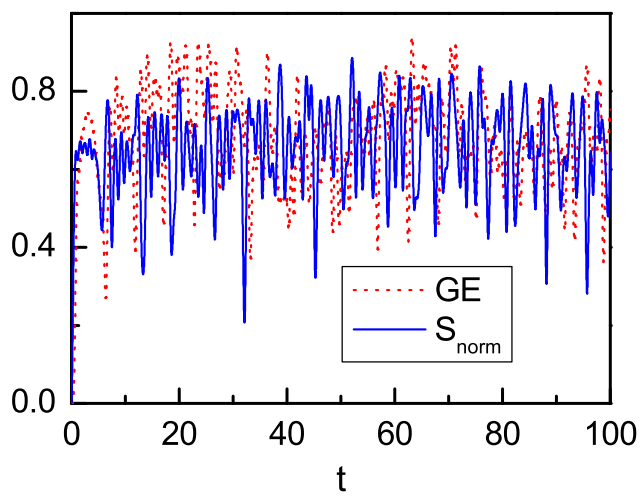

FIG. 3: (color online) Time dependence of average value of participation number $\left\langle N_{A B}\right\rangle$ calculated over all balanced bipartitions of the system for (a) non-integrable and (b) integrable model with parameters discussed in Fig. 1, Curves from top to bottom correspond to transverse field $h_{x}=1.0$ to 0.1 in decreasing steps of 0.1 . Within a small time window, $\left\langle N_{A B}\right\rangle \propto e^{A t}$ for relatively large $h_{x}$, as shown by an exponential fit (circles) for $h_{x}=0.8$ in both panels.

cribe the exponential growth of Wigner harmonics to the fast entanglement generation in the chain.

To better clarify this latter point, we have compared the time-dependence of $S(t)$ with the so-called "global entanglement" (denoted GE) [15]. In Ref. [16], it was shown that $G E$ is related to the averaged one-qubit purity, i.e.,

$$
G E=2\left(1-\frac{1}{N} \sum_{k=1}^{N} \operatorname{Tr}\left[\hat{\rho}_{k}^{2}\right]\right),
$$

where $\hat{\rho}_{k}$ is the density matrix of the $k$-th spin after tracing over all other spins in the system. $G E$ is the average bipartite entanglement over all possible bipartitions between a single qubit and the rest of the system. It is easy to see that $0 \leq G E \leq 1$. Values of $G E$ close to one indicate highly entangled many-body states. When a many-body state is not entangled, $G E$ equals to zero.

For the initial state $\left|\Psi_{\text {in }}\right\rangle=|\downarrow \downarrow \ldots \downarrow\rangle$, we present in Fig. 4 a comparison between $S$ and $G E$. To better visu-
FIG. 4: (color online) Comparison of the dynamics of normalized entropy $S_{\text {norm }}$ and global entanglement $G E$ for a chain of 10 spins with transverse field $h_{x}=0.8$. Panel (a) corresponds to the non-integrable model with longitudinal field $h_{z}=1.0$ and panel (b) corresponds to the integrable model. Here $S_{\text {norm }}$ is the entropy $S$ divided by its maximum value $N \ln 2$. A close correspondence between the dynamics of two measures is evident.

alize their similarities, we plot a normalized (to unity) entropy $S_{\text {norm }}=S / N \ln 2$. It is clearly observed that these two quantities show a high degree of resemblance in their time dependence. Their oscillating patterns are quite close and in some regimes they are almost on top of each other. The similarity between $S_{\text {norm }}$ and $G E$ constitutes strong evidence that our entropy measure $S(t)$, though originated from considerations of phase-space complexity, also reflects the degree of multipartite entanglement in many-body systems.

Though, in general, $G E$ may not distinguish between different classes of multipartite entangled states, it is an indicator of the critical point of, for instance, the quantum phase transition for the Ising chain in a transverse magnetic field [17]. Therefore, it is also interesting to investigate the behavior of $S(t)$ in the neighborhood of a quantum critical point which, for the transverse Ising chain in Eq. (16) with coupling strength $J=1$, is at $h_{x}=1$. In Fig. [5 we show the behavior of $S(t=0.5)$ 
(a)

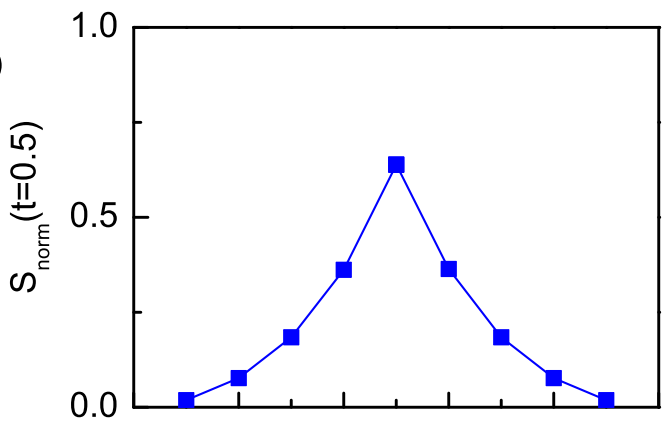

(b)

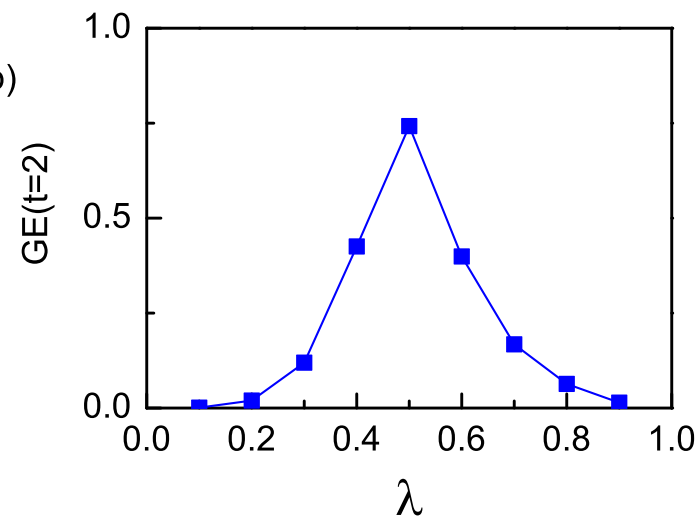

FIG. 5: (color online) Panel (a) shows $S(t=0.5)$ normalized by a factor of $N \ln 2$ as a function of the transverse field $h_{x}$ for a transverse Ising chain of 10 spins. Panel (b) shows the parallel results for $G E$ at $t=2.0$. Here $\lambda \equiv h_{x} /\left(J+h_{x}\right)$. Similar to $G E$, the complexity measure $S$ is seen to peak clearly at the critical point $\lambda=1 / 2$.

as well as $G E(t=2)$, as a function of $\lambda \equiv h_{x} /\left(J+h_{x}\right)$. Note that $S$ and $G E$ are plotted at different times because of their different saturation times. In addition, in our calculations of $S$ on the large-field side $(\lambda>1 / 2)$ the $x$ axis is used as the quantization axis of the basis states: The magnetic field term is dominant and correspondingly we define $\hat{H}^{(0)}=\sum_{i} h_{x} \hat{\sigma}_{i}^{x}, \hat{H}^{(1)}=J \sum_{i} \hat{\sigma}_{i}^{z} \hat{\sigma}_{i+1}^{z}$, and Schwinger bosons such that $\hat{\sigma}_{i}^{x}=\hat{a}_{i}^{\dagger} \hat{a}_{i}-\hat{b}_{i}^{\dagger} \hat{b}_{i}$. It is quite natural to consider as preferential basis the one associated with the dominant term in the Hamitonian: the $z$ basis when $\lambda \rightarrow 0$ and the $x$-basis when $\lambda \rightarrow 1$, and quantum phase transition corresponds to the switching from one preferential basis to the other. Consistent with the expectation that the quantum phase transition occurs at $\lambda=1 / 2$, Fig. [5(a) shows that $S_{\text {norm }}(t=0.5)$, a measure of the growth rate of the number of Wigner harmonics, exhibits a sharp peak at $\lambda=1 / 2$. The $\lambda$-dependence of $G E$ shown in Fig. [5) is analogous to what we observe in Fig. I5(a). This further demonstrates the close connection between our complexity measure $S(t)$ and the global en- tanglement $G E$ and, in particular, the role of many-body entanglement in the initial growth of $S(t)$.

Two additional aspects of $S(t)$ are in order. First, if we stick to the $z$ axis as the quantization axis of the basis states, then it is found that right after the critical point, $S(t)$ (if averaged over a time window to remove fluctuations) will show clear saturation behavior, which is in contrast to the monotonous increase of $S(t)$ before the critical point. Second, if we switch the quantization axis from $x$ to $z$ at other values of $\lambda$, then the value of $S(t=0.5)$ jumps discontinuously due to the change of the basis states. These additional results further suggest that the critical point for quantum phase transitions can be detected by $S(t)$.

Note that a different phase-space measure [18] has been used in the literature to detect quantum phase transitions [19]. However, such measure accounts for the extent at which the phase space is covered by the Husimi distribution and therefore it does not appear clear how to extend it to a suitable complexity measure for mixed states. In contrast, our measure which is based on the richness of the phase space structure rather than on phase-space coverage can be used for both pure and mixed quantum states. For instance, it could be used also to investigate thermal phase transitions.

\section{Wigner Harmonics, Chaos, and Thermalization}

Our results so far indicate that due to the dynamically generated many-body entanglement, the initial time-dependence of $S(t)$ does not reflect the peculiarity of quantum chaos in many-body quantum systems: it behaves similarly in integrable and non-integrable models. Note that this does not contradict with previous findings regarding rapid bipartite entanglement generation in classically chaotic systems with two degrees of freedom [20]. Indeed, in systems with two degrees of freedom, the Hilbert space is only a product of two subspaces and the quantum dynamics can only generate bi-partite entanglement. The rate of entanglement growth within such a fixed product of two subspaces is connected with the underlying classical dynamics. By contrast, in a manybody system such as our model used here the dynamics emanating from a local initial state is seen to explore more and more the tensor-product structure of the total Hilbert space and hence entangle more and more degrees of freedom during the time evolution. Our entropy measure $S(t)$ then is indicative of the growth rate of the number of degrees of freedom that have been entangled by the dynamics (a property absent in few-body systems).

Since the short-time behavior of $S(t)$ is seen to be unrelated to quantum chaos, we now examine the manifestation of quantum chaos in the long-time behavior of $S(t)$. This can be justified because after all, the peculiar spectral statistics of a quantum chaotic many-body system reflects the long-time properties of the system.

The time-dependence of $S(t)$ for a non-integrable 
(a)

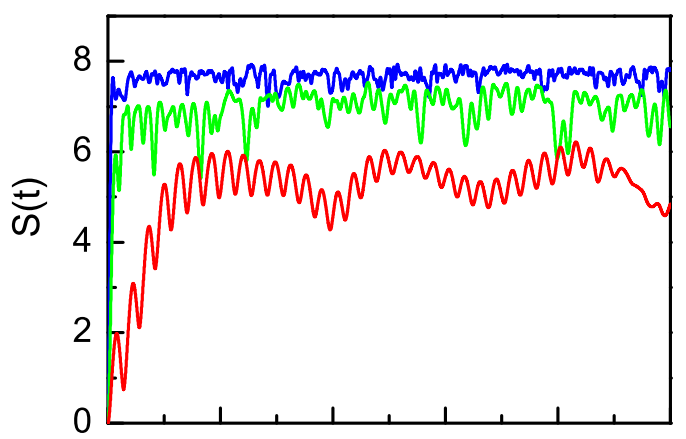

(b)

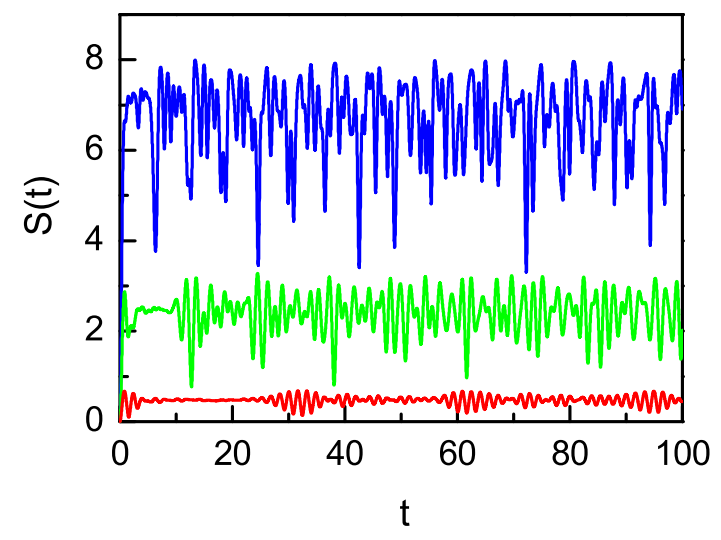

FIG. 6: (color online) Time evolution of entropy $S(t)$ for a chain of 12 spins with (a) non-integrable Hamiltonian in Eq. (15) for $h_{z}=1.0$ and (b) integrable Hamiltonian in Eq. (16). Curves from top to bottom corresponds to transverse field $h_{x}$ $=1.0,0.5$ and 0.2 . Note that the long term dynamics of the entropy in the two panels is qualitatively different.

model with the Hamiltonian given in Eq. (15) is shown in Fig. 6(a). The parallel result for an integrable model defined by Eq. (16) is shown in Fig. 6(b). In both cases, the time scale under study is now 20 times longer than that used in Fig. 1. It is seen that, in both panels (a) and (b), $S(t)$ initially quickly increases and then displays saturation with rich oscillating behavior. The saturation plateau of $S(t)$ increases as the value of the transverse field increases. Qualitatively, the saturation plateau can be attributed to an effective dimension of the Hilbert space that can be explored for a particular strength of the transverse field. To quantitatively describe this observation, we calculate the average value of the entropy from time $t_{1}=5$ to $t_{2}=100$ as $\bar{S}=\frac{1}{\tau} \int_{t_{1}}^{t_{2}} d t S(t)$, where $\tau=t_{2}-t_{1}$. The values of $t_{1}$ and $t_{2}$ are chosen so that the saturation plateau is reached before $t_{1}$ and $t_{2}$ is large enough to allow averaging over many oscillations of $S(t)$ in the time interval between $t_{1}$ and $t_{2}$. We have checked that other such choices of $t_{1}$ and $t_{2}$ do not affect any of our observations reported below. In Fig. 7, we plot $\bar{S}$ as a function of the transverse field, for the integrable and non-integrable models considered in Fig. 6. It is seen that, for small values of $h_{x}$, there is a difference between integrable and non-integrable dynamics. However, as the strength of the transverse field increases, this difference reduces. This is somewhat expected due to the abovediscussed many-body entanglement generation.

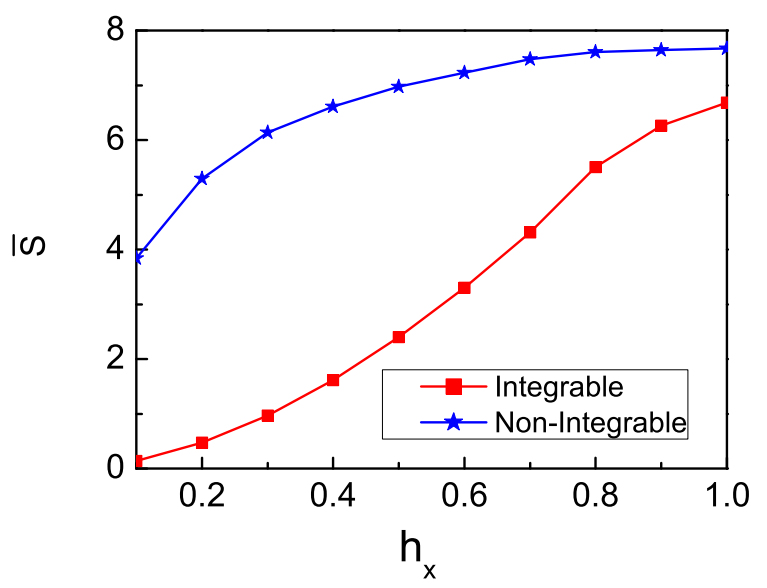

FIG. 7: (color online) Time averaged entropy, denoted $\bar{S}$, vs the strength of the transverse field $h_{x}$, for a chain of 12 spins. The non-integrable model corresponds to the Hamiltonian in Eq. 15 with $h_{z}=1.0$; the integrable model corresponds to the Hamiltonian in Eq. (16). The difference in $\bar{S}$ between integrable and non-integrable dynamics decreases with increasing $h_{x}$.

In order to distinguish between integrable and nonintegrable cases, we are thus forced to look into the oscillating behavior (rather than the average behavior) of $S(t)$. Indeed, from Fig. 6 one observes that in the nonintegrable case, the oscillation amplitude of $S(t)$ clearly decreases with the value of $h_{x}$. The oscillation pattern also becomes erratic as the system gets closer to the chaotic regime. By contrast, in the integrable case the opposite trend is observed. Regular and strong quantum revivals in $S(t)$ become more apparent as $h_{x}$ increases. To quantitatively describe this clear difference, we calculate the standard deviation of $S(t)$ around the mean value $\bar{S}$,

$$
\sigma[S]=\sqrt{\frac{1}{\tau} \int_{t_{1}}^{t_{2}} d t[S(t)-\bar{S}]^{2}} .
$$

The results are shown in Fig. 8, With increasing perturbation, the standard deviation $\sigma[S]$ increases and then saturates in the integrable model, so that the relative size $\sigma[S] / \bar{S}$ of fluctuations remains nearly constant. However, in the non-integrable model, the standard deviation $\sigma[S]$ and, more markedly, $\sigma[S] / \bar{S}$ decrease during the regularto-chaotic crossover (The same qualitative behavior is obtained when the number of the spins $N$ in the chain is 
varied). For $N=12, \sigma[S]$ in the integrable model with $h_{x}=1$ is around 0.878. By sharp contrast, in the nonintegrable case with $h_{x}=1, \sigma[S]=0.1495$, which is smaller than the first case by more than 5 times. This is a dramatic difference considering that the total number of spins in the chain is only 12 .

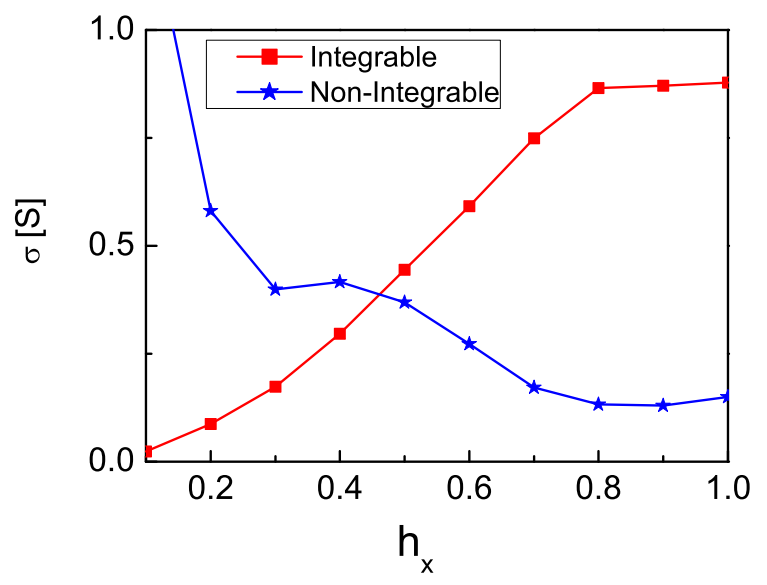

FIG. 8: (color online) Standard deviation $\sigma[S]$ in the entropy $S(t)$ vs the strength of the transverse field $h_{x}$ for a chain of 12 spins. Here the integrable and non-integrable models are the same as in Fig. 7. It is observed that as $h_{x}$ increases, the standard deviation generally decreases in the non-integrable model, but increases in the integrable model. For large values of $h_{x}, \sigma[S]$ for a non-integrable chain is much smaller than that for an integrable chain.

The large value of the standard deviation for the integrable model can be accounted for by the lack of thermalization. Indeed, our expectation is that the onset of chaos leads to internal dynamical thermalization 21], so that a statistical description is possible even though we have a closed, finite Hamiltonian system. Since the density of many-body energy levels grows exponentially with the number of particles, even a weak interaction between particles typically leads to a strong mixing on noninteracting many-body states, thus resulting in chaotic eigenstates. That is to say, the components of such eigenstates can be treated as random variables and therefore statistical methods can be applied to the description of local observables, in spite of the fact that close systems are under consideration. In such situation, fluctuations of the expectation values of local observables are small. On the contrary, in the integrable regime the lack of thermalization allows large fluctuations. To verify the above expectations, we have considered the Pauli operator $\hat{\sigma}^{x}$ (note that, due to translational invariance of model and initial condition, $\left\langle\hat{\sigma}_{i}^{x}\right\rangle(t)$ is independent of $i$ at any time $t)$. Observing the long term dynamics of the chain, we compute in the same way as in Eq. (19) for $S(t)$ the standard deviation $\sigma[X]$ of the $x$-polarization expecta- tion value $X(t) \equiv\left\langle\hat{\sigma}^{x}\right\rangle(t)$. Our results show that in the integrable model, the standard deviation increases with the transverse field whereas for the non-integrable case, the standard deviation decreases. This behavior, illustrated in Fig. 9, is qualitatively similar to the behavior of $S(t)$ shown in Fig. 8 . This shows that our complexity measure is related to the thermalization properties of the system.

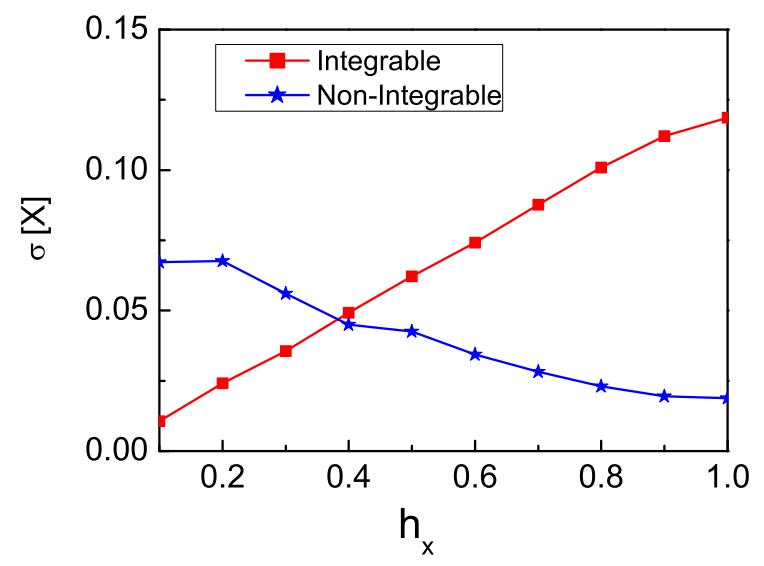

FIG. 9: (color online) Standard deviation of the $x$ polarization expectation value as a function of transverse field $h_{x}$ for a chain of 12 spins. Here the integrable and nonintegrable models are the same as in Fig. 7 Similar to the case of entropy $S(t)$, with increasing perturbation, the standard deviation $\sigma[X]$ decreases in the non-integrable model and increases in the integrable model.

\section{CONCLUSIONS}

In this paper, we propose an entropy measure $S(t)$ for many-body quantum-dynamical complexity, by extending the Wigner harmonics measure introduced in [2] [4] for single-particle quantum dynamics. The effectiveness of this measure is illustrated in the example of the Ising chain in a homogeneous tilted magnetic field. The Wigner harmonics entropy $S(t)$ exhibits an initial linear growth in both integrable and chaotic regimes, until saturation occurs due to the finite size of the Hilbert space. Therefore, in both integrable and chaotic regimes the number of harmonics of the Wigner function grows exponentially with time. In classical dynamics, an exponential growth of the number of harmonics of the classical phase-space distribution function implies chaotic dynamics. Therefore, the observed exponential growth of Wigner harmonics in the many-body quantum integrable regime must be attributed to a source of complexity absent in classical dynamics, that is, entanglement. We have numerically demonstrated the close connection be- 
tween our complexity measure $S(t)$ and multipartite entanglement, thus providing evidence that the initial linear growth of $S(t)$ has to be ascribed to multipartite entanglement generation. The Wigner harmonics measure $S(t)$ can also distinguish between integrable and chaotic many-body systems, by means of the size of long term fluctuations, which are smaller in the chaotic regime where a statistical description of the system is legitimate and the relative size of fluctuations drops when the system size increases.

The main advantage of the phase-space approach to complexity resides in its generality. At the classical limit, the harmonics of the phase-space distribution function reproduce the well-known notion of complexity based on local exponential instability [2]: the number of harmonics grows linearly for integrable systems and exponentially for chaotic systems. In single-particle quantum mechanics, an exponential growth of the number of harmonics is possible only up to the Ehrenfest time scale, after which the growth is at most linear [3]. Furthermore, the num- ber of harmonics of the Wigner function can be used to detect, in the time domain, the crossover from integrability to chaos [4]. For quantum many-body systems, the Wigner harmonics entropy measure $S(t)$ proposed in this paper signals the generation of multipartite entanglement and can be used to detect quantum phase transitions. In relation to other measures of complexity based on the efficiency of the best classical simulations of quantum systems [6], our approach has the advantage that it does not rely on a specific computational method like the time-dependent density-matrix renormalization group. Finally, we point out that, in contrast to other quantum phase-space approaches based on the moments of the Husimi function [18, 19], our complexity measure works equally well for either pure or mixed quantum states. Therefore, our measure could be studied in relation to mixed-state entanglement. This would be particularly interesting as mixed-state entanglement is at present not well understood and is the focus of ongoing research.
[1] J. Ford, Phys. Today 36, 40 (1983); V.M. Alekseev and M.V. Jacobson, Phys. Rep. 75, 287 (1981).

[2] J.B. Gong and P. Brumer, Phys. Rev. A 68, 062103 (2003); A. K. Pattanayak and P. Brumer, Phys. Rev. E 56, 5174 (1997).

[3] V. V. Sokolov, O. V. Zhirov, G. Benenti, and G. Casati, Phys. Rev. E 78, 046212 (2008).

[4] G. Benenti and G. Casati, Phys. Rev. E 79, 025201(R) (2009).

[5] R. Alicki and M. Fannes, Quantum Dynamical Systems (Oxford University Press, New York, 2001); T. Prosen, J. Phys. A 40, 7881 (2007); F. Benatti, Dynamics, Information and Complexity in Quantum Systems (Springer, 2009), and references therein.

[6] T. Prosen and M. Žnidarič Phys. Rev. E 75, 015202(R) (2007).

[7] P. Calabrese and J. Cardy, J. Stat. Mech. P04010 (2005); G. De Chiara, S. Montangero, P. Calabrese, and R. Fazio, J. Stat. Mech. P03001 (2006).

[8] V. Bargmann, Commun. Pure Appl. Math. 14, 187 (1961); R. J. Glauber, Phys. Rev. 1312766 (1963); G. S. Agarwal and E. Wolf, Phys. Rev. D 2, 2161 (1970); ibid. 2187.

[9] Note however, the second moment of the Fourier harmonics and the entropy measure $S(t)$ are not equivalent. For instance, in systems like the classical Arnold cat map, it turns out that the number of the Fourier harmonics is preserved in time but the second moment increases. See, for example, T. Prosen, preprint arXiv:1008.2419 [nlin.CD].

[10] J. Schwinger, Phys. Rev. 91, 728 (1953).

[11] S. Sachdev, Quantum Phase Transitions (Cambridge University Press, Cambridge, 1999).

[12] A. Auerbach, Interacting Electrons and Quantum Mag- netism (Springer-Verlag, New York, 1994).

[13] E. Lieb, T. Schultz, and D. Mattis, Ann. Phys. 16, 407 (1961).

[14] P. Facchi, G. Florio, and S. Pascazio, Phys. Rev. A 74, 042331 (2006).

[15] D. A. Meyer and N. R. Wallach, J. Math. Phys. 43, 4273 (2002).

[16] G. K. Brennen, Quantum Inf. Comput. 3, 619 (2003).

[17] T. R. de Oliveira, G. Rigolin, and M. C. de Oliveira, Phys. Rev. A 73, 010305(R) (2006).

[18] A. Sugita, J. Phys. A: Math. Gen. 36, 9081 (2003).

[19] S. Schenk and G.-L. Ingold, Phys. Rev. A 75, 022328 (2007).

[20] K. Furuya, M. C. Nemes, and G. Q. Pellegrino, Phys. Rev. Lett. 80, 5524 (1998); P. A. Miller and S. Sarkar, Phys. Rev. E 60, 1542 (1999); A. Lakshminarayan, Phys. Rev. E 64, 036207 (2001); A. Tanaka, H. Fujisaki, and T. Miyadera, Phys. Rev. E 66, 045201 (2002); J. B. Gong and P. Brumer, Phys. Rev. A 68, 022101 (2003); S. Ghose, P. M. Alsing, B. C. Sanders, and I.H. Deutsch, Phys. Rev. A 72, 014102 (2005)

[21] S. Åberg, Phys. Rev. Lett. 64, 3119 (1990); J. M. Deutsch, Phys. Rev. A 43, 2046 (1991); M. Srednicki, Phys. Rev. E 50, 888 (1994); V. V. Flambaum and F. M. Izrailev, Phys. Rev. E 56, 5144 (1997); Ph. Jacquod and D. L. Shepelyansky, Phys. Rev. Lett. 79, 1837 (1997); G. Benenti, G. Casati, and D. L. Shepelyansky, Eur. Phys. J. D 17, 265 (2001); C. Kollath, A. M. Läuchli, and E. Altman, Phys. Rev. Lett. 98, 180601 (2007); S. R. Manmana, S. Wessel, R. M. Noack, and A. Muramatsu, Phys. Rev. Lett. 98, 210405 (2007); M. Rigol, V. Dunjko, and M. Olshanii, Nature 452, 854 (2008). 